\title{
Novel Medication Supply Model Guarantees Adequate Management and High Adherence to Polypharmacy in Older Opioid Users - Preliminary Results with Outpatients
}

\author{
Isabelle Arnet ${ }^{1 *}$, Samuel S Allemann ${ }^{1}$, Kenneth M Dürsteler ${ }^{2}$, Johannes Strasser ${ }^{2}$, Marc Vogel $^{2}$ and Kurt E Hersberger ${ }^{1}$ \\ ${ }^{1}$ Pharmaceutical Care Research Group, University of Basel, Switzerland \\ ${ }^{2}$ Division of Substance Use Disorders, Psychiatric University Clinics of Basel, Switzerland \\ "Corresponding author: Isabelle Arnet, Pharmaceutical Care Research Group, University of Basel, Switzerland, E-mail: isabelle.arnet@unibas.ch
}

Received date: March 01, 2017; Accepted date: March 22, 2017; Published date: March 24, 2017

Copyright: ( 2017 Yong-chang et al. This is an open-access article distributed under the terms of the Creative Commons Attribution License, which permits unrestricted use, distribution, and reproduction in any medium, provided the original author and source are credited.

\begin{abstract}
Background: Life expectancy of older drug users has increased, primarily thanks to opioid agonist treatment (OAT). Nursing homes are often not adapted to accommodate patients with substance use disorders. Although care and adherence to polypharmacy in older opioid users need considerable resources e.g., daily visits to an outpatient clinic, outpatient treatment and surveillance are provided as long as possible. We developed a novel medication supply model with an electronic dispenser of pre-packed medications located at patient home, after allowing for law requirements concerning the dispensing of opioids, and present preliminary results from three illustrative outpatients.

Methods: The community pharmacy provided unit-of-dose pouches with all solid oral medications directly to patient home. Opioids for substitution were obtained at the addiction clinic in at least weekly intervals, otherwise in the pouches. The pouches were loaded into a lockable, remote-controlled medication management aid that was programmed according to the patient's medication schedule. The dispenser reminds patients with acoustic alerts to take their medication and records dates and times of medication retrievals. It automatically sends an alert if a patient misses to retrieve a dose.
\end{abstract}

Results: Our three outpatients used the electronic dispenser during 659,118 and 61 days, with a total of 5,9 , and 18 pills to take daily at 1,3 and 5 intake times, respectively. The majority of the doses were taken on the preset time $(94 \%, 68.2 \%$ and $73.7 \%)$ or deliberately in advance (pocket dose). Clinical benefits were initiation and maintenance of a therapy for dementia over 18 months and suppression of HIV viral load over 1.8 years (patient 1), prevention of further dose escalation of pain medication (patient 2) and release of prompts to initiate the existential task of cooking (patient 3).

Conclusion: Our novel supply model allows adequate implementation and persistence of complex treatments with outpatients. Clinical outcomes improved, patients and caregivers were satisfied, and resources were saved.

Keywords: Electronic medication dispenser; Electronic adherence monitoring; Medication management aid; Polypharmacy; Opioid agonist treatment; Interprofessional collaboration; Solution; Unit-ofdose pouches

\section{Introduction}

Adherence is defined as "the process by which patients take their medications as prescribed, composed of initiation, implementation and discontinuation" [1]. Persistence describes the time length between the first and the last dose i.e., between initiation and discontinuation. However, being persistent does not imply that the actual dosing corresponds to the prescribed regimen. Modern medicine has developed a multitude of evidence-based treatments for many chronic diseases. As a result, the prescription of several medications to treat one or more conditions in the same patient has become increasingly common, especially in the elderly $[2,3]$. In the United States and Europe, more than $50 \%$ of elderly patients use more than 5 prescription medications $[4,5]$. With the demographic shift toward older age and the concurrent increase in morbidity, the prevalence of polypharmacy will likely increase in the future, representing an additional factor contributing to non-adherence [6].

Along with the aging of the general population, the number of older users of illicit substances (or older drug users) is also growing in Europe [7] and in Switzerland [8]. Owing to harm reduction services (such as needle and syringe programs), significant improvements in availability, access and quality of treatment (such as highly active antiretroviral therapy (HAART) for HIV/AIDS) and opioid agonist treatment (OAT), life expectancy has increased in older drug users. Consequently, they may be affected on the one hand by progressive conditions such as HIV or chronic hepatitis $\mathrm{C}$ virus (HCV) infection that may take decades to cause significant illness or death, and on the other hand by age-related comorbidities. Moreover, older drug users are likely to suffer from neurocognitive deficits. This may result from the accelerated aging process or from mental health effects of polysubstance use, overdoses and infections [9]. Finally, older drug users are often socially excluded or marginalised. Thus, support from family or friends is mostly inexistent or unreliable. OAT is the main evidence-based treatment for heroin dependence and is the treatment of first choice in many international guidelines (WHO, NICE). It is 
Citation: Arnet I, Allemann SS, Dürsteler KM, Strasser J, Vogel M, et al. (2017) Novel Medication Supply Model Guarantees Adequate Management and High Adherence to Polypharmacy in Older Opioid Users - Preliminary Results with Outpatients. J Aging Sci 5: 176. doi:10.4172/2329-8847.1000176

Page 2 of 7

efficacious, cost-effective and well tolerated [10]. Currently, traditional OAT with liquid Methadone is increasingly being replaced by solid formulations such as Buprenorphine or sustained-release Morphine [11]. Besides OAT, many older drug users take several long-term medications. These findings have multiple implications, in particular with respect to the often complex multiple medication (polypharmacy). Thus, older patients on OAT may be particular prone to errors in medication intake. Correct implementation of treatment is crucial for opioid dependent patients, not only to guarantee optimal treatment of the underlying illness, but also to minimize dependence and withdrawal symptoms. In addition, the treatment of chronic viral conditions such as HIV or HCV is expensive and require adherence rates of more than $95 \%$ to be effective [12], forcing health care professionals to develop strategies that guarantee medication intake, e.g. with outreach and low-threshold specialized outpatient centres and direct observed therapy (DOT). As a consequence, treatment of older drug users evolves into intensive and resource consuming care. Thus, new solutions for the achievement of sustainable and affordable care are needed.

Finally, with approximatively 2 million people being addicted to prescription opioids for chronic pain in the United States, the magnitude of opioid use and misuse in the US has been recently described as epidemic [13]. Because substance use disorders such as opioid dependence often coincide with multiple risk factors for medication non-adherence (such as unemployment or low social support), health care professionals are called to change their practice. In this spirit, the Surgeon General of the United States advocated for treatment programs including monitoring of medicine use [13].
To conclude, alternative supply models are needed to assist patients with their medication management and to optimize adherence, including chronic opioid users for any reason. Adherence management is defined as "the process of monitoring and supporting patients' adherence to medications by health care systems, providers, patients, and their social networks" [1]. Thus, management of adherence begins with the monitoring of said behaviour, and flows into a targeted intervention to improve adherence, if needed. We developed a novel medication supply model with interprofessional collaboration between an outpatient addiction clinic and a community pharmacy in Basel, Switzerland, with the aim of guaranteeing adherence to polypharmacy by using an electronic dispenser of pre-packed medications located at patient home. We tested the feasibility of our novel medication supply model with three illustrative outpatients and present preliminary results.

\section{Methods}

\section{Remote-controlled electronic monitoring with pre-packed unit-of-dose pouches}

Solid oral prescription medications are repacked into unit-of-dose pouches (Automatic Tablet Dispensing and Packaging System, Desk Type JV-30DE, HD-Medi, Germany). Each pouch is imprinted with the patient's data (name, date of birth) and medication data (number, name, date and time for intake, color, shape of the medication; Figure $1)$.
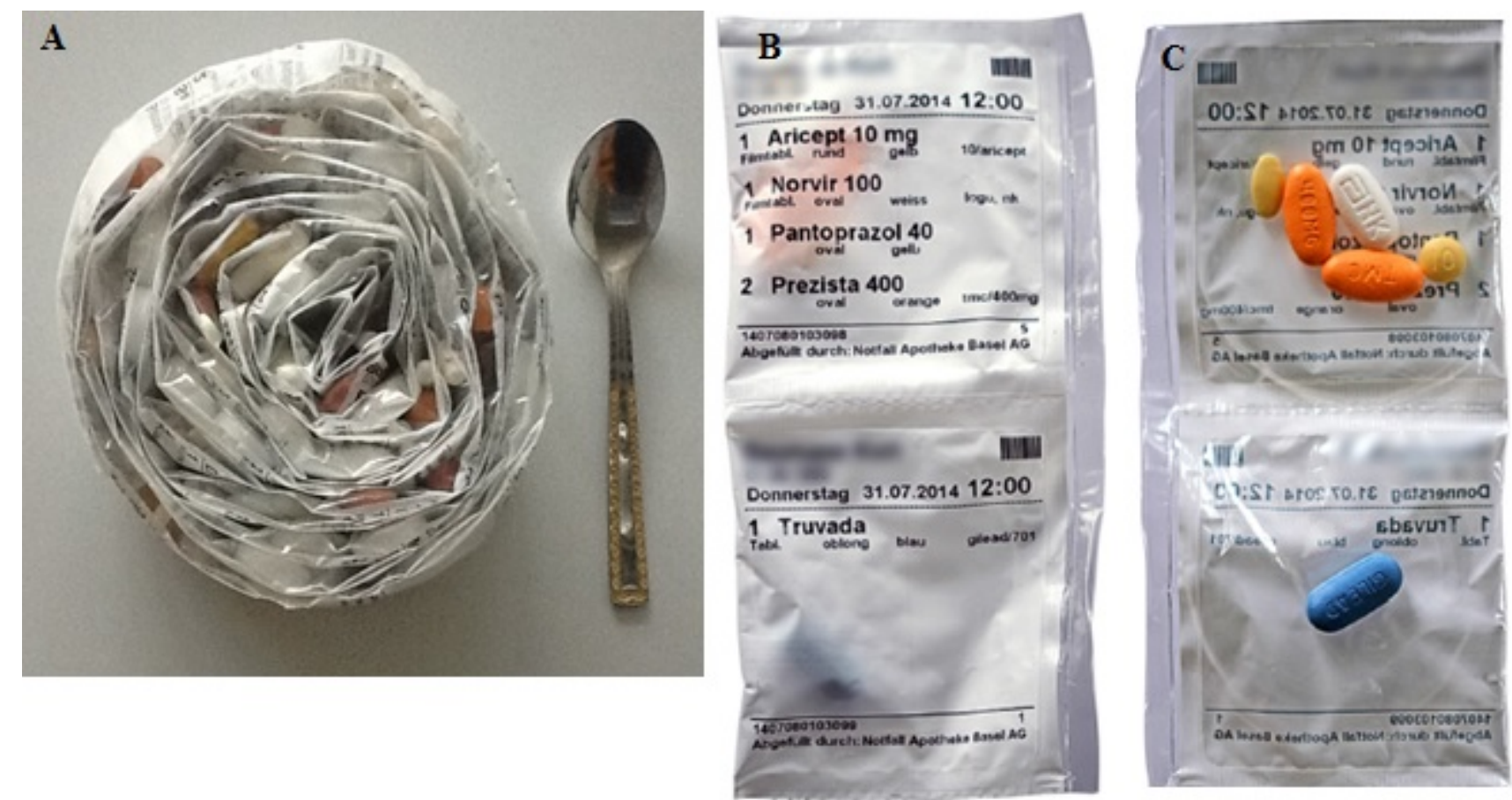

Figure 1: Unit-of-dose pouches with prepacked oral solid medication as roll (A), two pouches from front-side (B) and back-side (C). Note: patient's name and date of birth were concealed for privacy reasons.

Rolls of pouches for 1-3 weeks are placed in a remote controlled, electronic medication management aid reminding the patients with acoustic alerts to take their medication (Medido ${ }^{\circ}$, Innospense $\mathrm{BV}$, Netherlands; Figure 2). 
Citation: Arnet I, Allemann SS, Dürsteler KM, Strasser J, Vogel M, et al. (2017) Novel Medication Supply Model Guarantees Adequate Management and High Adherence to Polypharmacy in Older Opioid Users - Preliminary Results with Outpatients. J Aging Sci 5: 176. doi:10.4172/2329-8847.1000176

Page 3 of 7

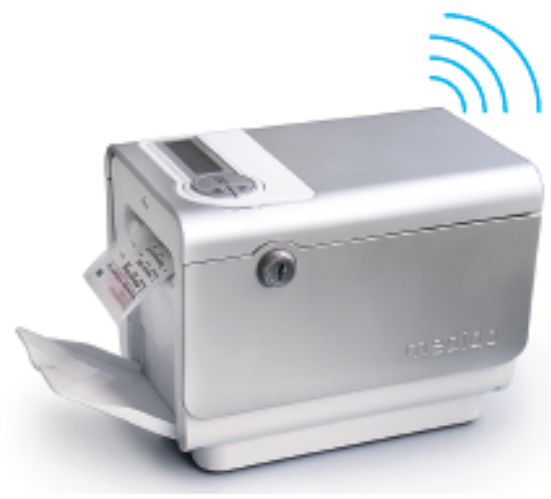

Figure 2: Remote-controlled, electronic medication management aid, medido, used in this study to dispense the unit-of-dose pouches (Height $\times$ Width $\times$ Length: $140 \mathrm{~mm} \times 140 \mathrm{~mm} \times 225 \mathrm{~mm}$; Weight: $1.48 \mathrm{~g})$.
A web-based application allows to set the time of dispense individually according to participants' preferences. Pushing the OKbutton stops the alarm and delivers the pouches with the pre-packaged medication. A sensor in the dispenser registers a barcode printed on the top of each pouch and cuts the pouches accordingly. Date and time of delivery are simultaneously transmitted to a secure server with GPRS-technology. Doses can be delivered ahead of schedule (so-called pocket-doses) by pushing the OK-button for 5 seconds. This feature enables patient mobility i.e., to be outside of home during intake times. The dispenser is installed at the patient' home and patient is orally instructed about its proper use. A written manual is given including a telephone hotline number in case of technical problems with the dispenser. A former qualitative analysis of the dispenser suggested that patients with time-sensitive medication regimens could benefit best [14]. Further, a similar type of electronic dispenser has been considered safe to provide OAT [15].

\section{Novel supply model}

Core element of the novel supply model is the respect of existing law requirements concerning opioid dispensing, and the supply of the entire co-medication at the patients' home with an electronic guardian.

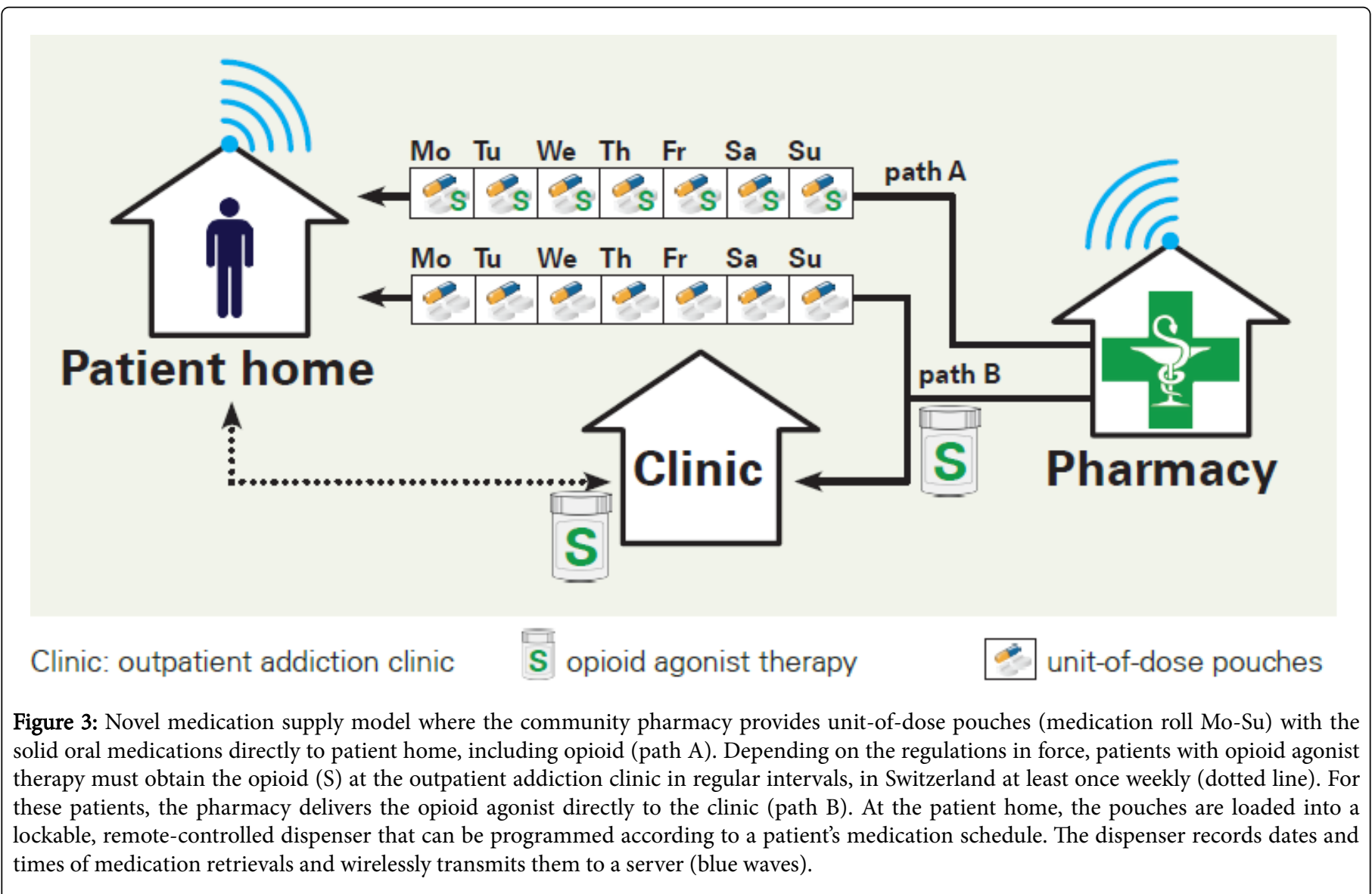

Patients obtain the opioid either for pain treatment together with all medications through official pharmacy services (Figure 3, path A) or for substitution from the addiction clinic in regular intervals, at least once weekly according to Swiss law (Figure 3, path B). After having performed a medication review, the community pharmacy determines an appropriate treatment schedule with the patient, and provides unit- of-dose pouches with all solid oral medications directly to patient home, either with (path A) or without (path B) opioid. 
Citation: Arnet I, Allemann SS, Dürsteler KM, Strasser J, Vogel M, et al. (2017) Novel Medication Supply Model Guarantees Adequate Management and High Adherence to Polypharmacy in Older Opioid Users - Preliminary Results with Outpatients. J Aging Sci 5: 176. doi:10.4172/2329-8847.1000176

Page 4 of 7

\section{Monitoring-induced actions}

If a patient misses to retrieve a dose from the dispenser after a predefined time of intake (in our study the grace period was preset at 75 minutes) or in case of malfunctioning, the dispenser automatically sends an alert. In our study, we selected alerts to be sent via short message service (SMS) to the hotline number that was operated by a pharmacist of the research group (SA or IA) during weekdays and by the Emergency Pharmacy during weekends and public holidays. The person in charge of the hotline then contacts the patient by phone call, inquires the situation and acts accordingly.

The password-protected, web-based application can be consulted at any time and indicates with a color code how the pouches have been dispensed (Figure 4). In case of a technical problem, the pharmacist can either resolve it by remote action or with a visit at the patient home. In any case, he makes sure that medication has been taken.

\section{Weekly schedule}

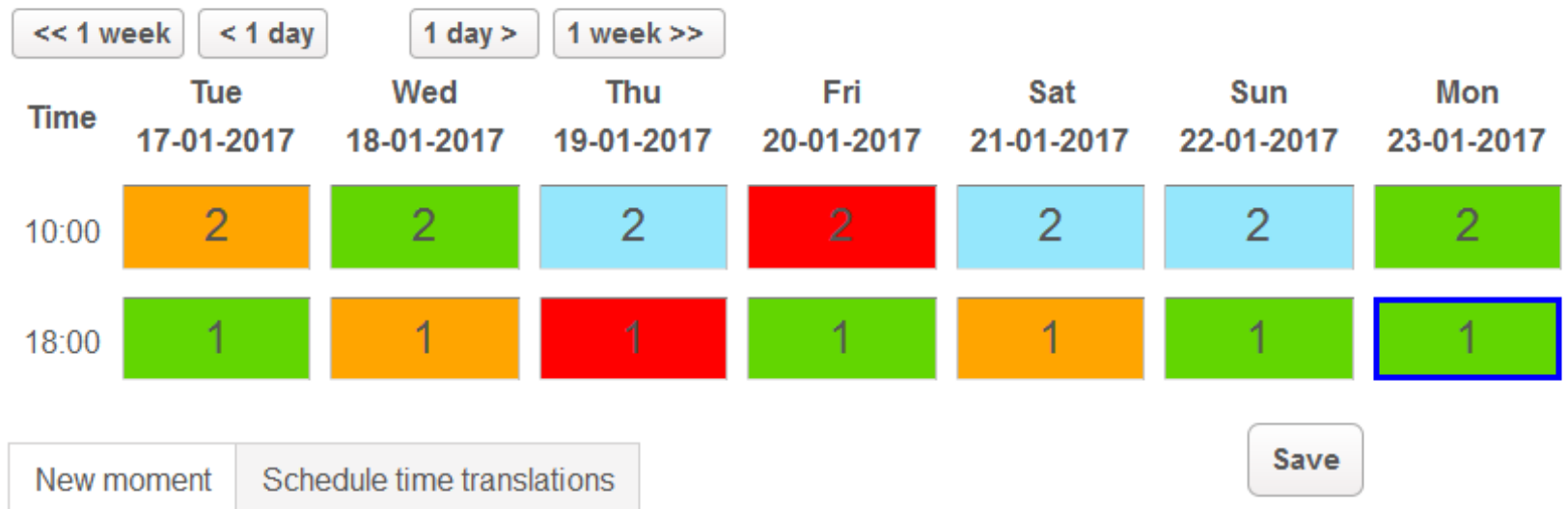

\section{Add a new moment (hhm0):}

Add moment

Figure 4: Web-based application of medido ${ }^{\circ}$ with colour coded retrievals of pouches during one week (January $17^{\text {th }}$ to $23^{\text {th }}, 2017$ ) for a patient with a twice daily intake of two pouches at $10 \mathrm{am}(10: 00)$ and one pouch at $6 \mathrm{pm}$ (18:00). A dispense time before the predefined time is indicated in orange (pocket-dose); a dispensing within a preset grace period (here 75 minutes) after the predefined time of intake is indicated in green (regular dose); a dispensing after 75 minutes is blue (late dose); and a forgotten dispense is red (missed dose).

\section{Patient selection}

We recruited three patients from an outpatient addiction clinic (Division of Substance Use Disorders, Psychiatric University Clinics of Basel) with different reasons for opioid treatment. All have been obtaining all medications including opioids from the outpatient clinic for years. Because of unmet clinical goals (i.e., flares of HIV viral load for patient 1; inadequate pain relief despite dose escalation for patient 2; malnutrition and hypovitaminosis for patient 3), conventional care and adherence to medication were questioned. Repetitively missed appointments at the clinic, mostly due to impaired mobility, impeded daily medication dispensing or daily direct observed therapy. Thus, the situation remained unsatisfactorily for the health care professionals. Our patients received no or little support from family or friends. During visits at the clinic, they admitted to have frequently missed medication intakes during the past 4 weeks. They accepted the offer of their caregivers to obtain medications with the novel supply model.
All three patients were unemployed, lived independently in social housing either alone (patient 3 ) or with a friend (patient 1) or a grandchild (patient 2) in Basel, a mid-size city in Switzerland. Patient 1 was HIV-positive since 25 years consecutive to intravenous drug abuse and joined a Methadone program that was switched in 2013 to $1,200 \mathrm{mg}$ long-acting Morphine daily (6 tablets) consecutive to a long QTsyndrome.

Patient 2 had immobilizing chronic pain treated with long-acting morphine $(1,000 \mathrm{mg})$ and various co-analgesics. She was hospitalized two weeks after entering the study, and entered stationary psychiatric rehabilitation, where the dispenser was not in use. After her return home, she resumed medication dispense from the electronic dispenser.

Patient 3 experienced malnutrition and hypovitaminosis after partial gastric resection due to obesity. She had developed severe lymphedema in her legs after an infection, which lead to sustained impairment of her mobility. She lacked impetus and often slept in, disturbing her daily routine. 
Citation: Arnet I, Allemann SS, Dürsteler KM, Strasser J, Vogel M, et al. (2017) Novel Medication Supply Model Guarantees Adequate Management and High Adherence to Polypharmacy in Older Opioid Users - Preliminary Results with Outpatients. J Aging Sci 5: 176. doi:10.4172/2329-8847.1000176

Page 5 of 7

\section{Results}

All three patients understood that the electronic dispenser would bring a benefit for their medication management. They accepted the novel medication supply model and gave written consent. They were aware of the electronic monitoring and the automatic alarms in case of missed dispense. The dispensers were installed in the kitchen (patient 1 and 3), or in the living room (patient 2). Dispensing times were scheduled in line with consistent habits of daily life i.e., $12 \mathrm{pm}$ for patient 1 (first meal of the day), and according to meals and watching TV for patient 2 and 3 (see Table 1). Every 3 weeks, medications were repackaged according to the current treatment plan and the dispenser was refilled during a pre-scheduled visit at the patient home. On this occasion, patients were asked about their well-being, difficulties with the use of the dispenser, and whether they had taken their medication immediately after retrieval of the pouches from the dispenser. Patients declared to be satisfied with the novel supply model, and the few technical problems did not jeopardize medication intake. Errors during the dispensing of the pouches occurred predominantly at the end of a medication roll, and did not require any intervention.

\begin{tabular}{|c|c|c|c|}
\hline & Patient 1 & Patient 2 & Patient 3 \\
\hline Age at (study entry) & 50 (July 2, 2013) & 68 (Feb 25, 2015) & 36 (July 17,2015 ) \\
\hline Main diagnoses & $\begin{array}{l}\text { HIV, HCV, liver cirrhosis, } \\
\text { beginning Alzheimer's disease }\end{array}$ & $\begin{array}{l}\text { polyarthrosis, recurrent depressive disorder, } \\
\text { Basedow-Graves disease, hypertensive } \\
\text { heart failure }\end{array}$ & malnutrition, hypovitaminosis \\
\hline $\begin{array}{l}\text { Number of medicines (number of } \\
\text { pills) }\end{array}$ & $5(5)$ & $13(18)$ & $7(9)$ \\
\hline Number of intake times & 1 & 5 & 3 \\
\hline $\begin{array}{l}\text { Number of medications in pouches } \\
\text { @ preset dispense time : name and } \\
\text { strength of medication }\end{array}$ & 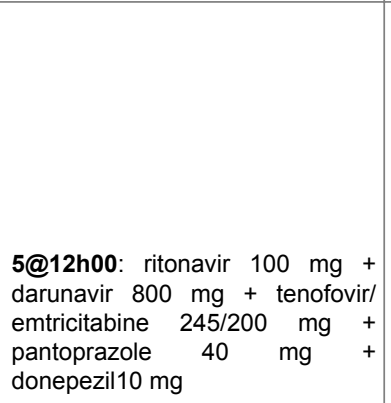 & 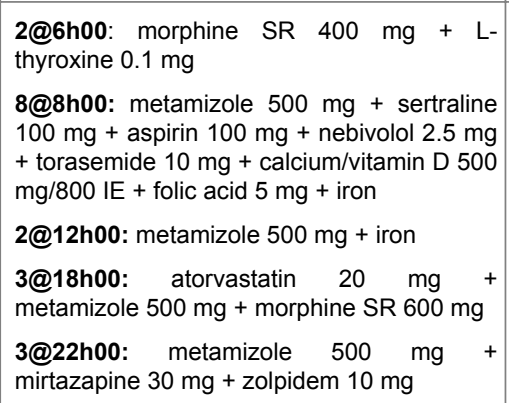 & $\begin{array}{l}\text { 5@10h00: multivitamins + calcium/vitamin } \\
\text { D } 500 \mathrm{mg} / 800 \mathrm{IE}+\text { zinc + pregabalin } 150 \\
\mathrm{mg} \\
\text { 2@12h00: calcium/vitamin D } 500 \mathrm{mg} / 800 \\
\mathrm{IE}+\text { pregabalin } 150 \mathrm{mg} \\
\begin{array}{l}\text { 2@18h00: quetiapine } \quad 150 \quad \mathrm{mg} \quad+ \\
\text { trimipramine } 100 \mathrm{mg}+\text { diazepam } 40 \mathrm{mg}\end{array}\end{array}$ \\
\hline Opioid dispense & At clinic weekly (not monitored) & In pouch & At clinic weekly (not monitored) \\
\hline Days of follow-up (study end) & 659 (Apr 2, 2015) & $61($ Apr 28, 2015) & 118 (Nov 11, 2015) \\
\hline Number of rolls replacement & 31 & 7 & 13 \\
\hline Days with electronic monitoring & 655 & 45 & 118 \\
\hline Regular dose (green) & $615(94.0 \%)$ & $98(73.7 \%)$ & $281(68.2 \%)$ \\
\hline Pocket dose (orange) & $1(0.2 \%)$ & $29(21.8 \%)$ & $121(29.4 \%)$ \\
\hline Late dispense (blue) & $8(1.2 \%)$ & $2(1.5 \%)$ & 0 \\
\hline Missed doses (red) & $4(0.6 \%)$ & $4(3 \%)$ & $10(2.4 \%)$ \\
\hline
\end{tabular}

Table 1: Characteristics of the 3 illustrative patients with number of pills, number of pouches and preset time of intakes. Regular dose: the doses were dispensed within 75 minute after the preset intake time; Pocket dose: the doses were dispensed before the preset intake time; Late dispense: the doses were dispensed later than 75 minute after the preset intake time; Missed doses: the doses were dispensed remotely after a pharmacist intervention. The colours correspond to the codes used in Figure 4.

For patient 1 , the use of the electronic dispenser enabled to initiate a therapy for dementia and to maintain it over 18 months, to obtain constant suppression of viral load and rising of CD4 cells over 1.8 years and to stay in his housing. According to his care givers, the novel supply model delayed patient's first admission to a nursing home by approximately 1 year. For patient 2, the electronic dispenser assured the timely and correct intake of her pain medication and prevented further dose escalations. The remote monitoring made daily visits to the clinic obsolete and allowed her to stay at her home, according to her immobility. For patient 3 , the dispenser added a clear structure to her day and provided the necessary prompts to take her medications. Subsequently, she used the medication alarms as reminders to initiate other tasks e.g., preparing a meal. As a result, her vitamin levels improved.

Dispensing the pouch before the predefined time (pocket dose) occurred approximatively for $25 \%$ of the doses for two patients. This corresponds to a phase of psychiatric rehabilitation program for 
patient 2 where she regularly retrieved pocket-doses in the morning before entering the clinic. Patient 3 started the study with 4 prescheduled times $(10 \mathrm{~h} 00,12 \mathrm{~h} 00,18 \mathrm{~h} 00$ and $20 \mathrm{~h} 00)$ and would spend most of the time at home. After 2 months, the latest time of intake was eliminated because the beeping in the evening was inconvenient, especially when friends would come to her place. Thus, what was recorded as pocket dose was in fact a deliberate dose.

\section{Discussion}

Existing nursing homes or home care services are scarce and expensive, and often not adapted to accommodate patients with substance use disorders such as older opioid users. Therefore, outpatient treatment and surveillance are provided as long as possible. However, considerable resources in infrastructure and staff are needed to guarantee care and adherence to polypharmacy in an aging population with opioid agonist treatment, such as daily visits to an outpatient clinic. Thus, new supply models and innovative solutions are needed that combine provision of care and optimization of medication management compatible with opioid treatment. In this regard, electronic dispensers of prepacked polypharmacy offer a unique opportunity. They allow adequate implementation and persistence of a complex treatment with patients staying at home, and rely on a system of alerts in case of problems.

In our study, follow-up times differed from case to case for various reasons, but not attrition. Patient 1 refused to quit the study after its official completion (6 months) predominantly because of the sheltered situation of a safe medication dispensing at home that was made possible by the dispenser. Ethical considerations forced the investigators to maintain the dispenser and the service in place until the patient entered a nursing home. Patient 2 deceased after 14 weeks (death unrelated to the study). Patient 3 finished the study after its official duration and could maintain the gained structured living habits. Thus, our intervention with remote technology has the potential to improve adherence to polypharmacy and to engrave new behaviours into daily habits. However, some patients may need the service forever.

Several major benefits are obtained with multiple medication supplied in unit-of-dose pouches. For example, changes in treatment are implemented without disturbances for the patient, especially when the new medication can be added to a pre-existing pouch; up-titration is feasible without errors, such as weekly dose increases of a medication for dementia; initiation of preventive and irregular treatment is guaranteed without errors, such as prophylaxis of Pneumocystis carinii in HIV treatment with one tablet three time per week (sulmamethoxazole/trimethoprime $800 \mathrm{mg} / 160 \mathrm{mg}$ Monday, Wednesday and Friday); and adaptation of the content of the pouches is possible without modifying the intake habits. Larger trials with similar devices suggest an improvement of adherence and clinical outcomes for patients with such diverse conditions as kidney transplantation [16] or schizophrenia [17]. However, study duration was mostly short and implementation of dosing regimens was not a main focus of interest. With our proposed supply model, clinical outcomes improved, patients and caregivers were satisfied, and resources were saved. Further, the number of on-site visits decreased, leaving the remaining visits entirely for discussion about current findings, and not about medication supply.

Mathematically, the novel supply model generated more costs than standard care, predominantly due to the addition of a dispenser, a hotline and pharmacists' work. The dispenser we used is not routinely available in Switzerland and the costs remain high at the moment. However, on the long term, costs might be saved for several reasons. First, the market price of the device after commercialisation might decrease, and a hotline might be integrated as part of the service. Second, pharmacists' work would be saved when patients would collect the rolls of medication at the clinic during a routine visit and refill the dispenser by themselves. Further, the number of visits to the clinic may decrease, in the extreme cases from several times daily (for direct observed therapy) to once weekly (as required by law for OAT). Thus, this increased independence might enable younger patients to pursue paid employment. Finally, postponement of assisted living may save large amounts of money to society.

\section{Conclusion}

Electronic medication management aids offer a sustainable solution for integrated care with an interprofessional approach (hospital or specialized clinic and community pharmacy) and can assure adequate implementation of and persistence with complex oral treatment. Electronic devices with prepacked unit-of-dose medications may ensure correct dosing regimens for patients with polypharmacy including opioid for any reasons. On the long term, the avoidance of more expensive alternatives such as direct observed therapy or nursing homes might offset the additional costs generated by our novel supply model.

\section{References}

1. Vrijens B, De Geest S, Hughes DA, Przemyslaw K, Demonceau J, et al (2012) A new taxonomy for describing and defining adherence to medications. Br J Clin Pharmacol 73: 691-705.

2. Guthrie B, Makubate B, Hernandez-Santiago V, Dreischulte T (2015) The rising tide of polypharmacy and drug-drug interactions: population database analysis 1995-2010. BMC Med 13: 74.

3. Nobili A, Franchi C, Pasina L, Tettamanti M, Baviera M, et al. (2011) Drug utilization and polypharmacy in an Italian elderly population: the EPIFARM-elderly project. Pharmacoepidemiol Drug Saf 20: 488-496.

4. Fialová D, Topinková E, Gambassi G, Finne-Soveri H, Jónsson P, et al. (2005) Potentially inappropriate medication use among elderly home care patients in Europe. JAMA 293: 1348-1358.

5. Kaufman DW, Kelly JP, Rosenberg L, Anderson TE, Mitchell AA (2002) Recent patterns of medication use in the ambulatory adult population of the United States: the Slone survey. JAMA 287: 337-344.

6. Hughes CM, Cadogan CA, Patton D, Ryan CA (2016) Pharmaceutical strategies towards optimising polypharmacy in older people. Int J Pharm 512: 360-365.

7. European Monitoring Centre for Drugs and Drug Addiction (2010) Treatment and care for older drug users. Luxembourg, Publications Office of the European Union.

8. Dursteler-MacFarland KM, Vogel M, Wiesbeck GA, Petitjean SA (2011) There is no age limit for methadone: a retrospective cohort study. Subst Abuse Treat Prev Policy 6: 9.

9. Rosen D, Hunsaker A, Albert SM, Cornelius JR, Reynolds Iii CF (2011) Characteristics and consequences of heroin use among older adults in the United States: A review of the literature, treatment implications, and recommendations for further research. Addict Behav 36: 279-285.

10. Ward J, Hall W, Mattick RP (1999) Role of maintenance treatment in opioid dependence. Lancet 353: 221-226.

11. Hämmig R, Köhler W, Bonorden-Kleij K, Weber B, Lebentrau K, et al. (2014) Safety and tolerability of slow-release oral morphine versus methadone in the treatment of opioid dependence. J Subst Abuse Treat 47: $275-281$ 
Citation: Arnet I, Allemann SS, Dürsteler KM, Strasser J, Vogel M, et al. (2017) Novel Medication Supply Model Guarantees Adequate Management and High Adherence to Polypharmacy in Older Opioid Users - Preliminary Results with Outpatients. J Aging Sci 5: 176. doi:10.4172/2329-8847.1000176

Page 7 of 7

12. Lima VD, Harrigan R, Murray M, Moore DM, Wood E, et al. (2008) Differential impact of adherence on long-term treatment response among naive HIV-infected individuals. AIDS 22: 2371-2380.

13. Murthy VH (2016) Ending the opioid epidemic - a call to action. New Engl J Med 375: 2413-2415.

14. Allemann S, Arnet I, Hersberger K (2015) Patient views on an electronic dispensing device for prepackaged polypharmacy: a qualitative assessment in an ambulatory setting. Integr Pharm Res Pract 2015: 167-174.

15. Uosukainen H, Pentikainen H, Tacke U (2013) The effect of an electronic medicine dispenser on diversion of buprenorphine-naloxone-experience from a medium-sized Finnish city. J Subst Abuse Treat 45: 143-147.
16. Henriksson J, Tyden G, Höijer J, Wadström J (2016) A prospective randomized trial on the effect of using an electronic monitoring drug dispensing device to improve adherence and compliance. Transplantation 100: 203-209.

17. Stip E, Vincent P, Sablier J, Guevremont C, Zhornitsky S, et al. (2013) A randomized controlled trial with a Canadian electronic pill dispenser used to measure and improve medication adherence in patients with schizophrenia. Front Pharmacol 4: 100. 